\title{
Clinical exome sequencing for cerebellar ataxia and spastic paraplegia uncovers novel gene-disease associations and unanticipated rare disorders
}

Bart P van de Warrenburg, Meyke I Schouten, Susanne T de Bot, Sascha Vermeer, Rowdy Meijer, Maartje Pennings, Christian Gilissen, Michèl AAP Willemsen, Hans Scheffer and Erik-Jan Kamsteeg

European Journal of Human Genetics (2017) 25, 393; doi:10.1038/ejhg.2016.168

Correction to: European Journal of Human Genetics (2016) 24, 1460-1466; doi:10.1038/ejhg.2016.42; published online 11 May 2016

Since publication, the authors have noticed that they had reported a sibship with autosomal recessive hereditary spastic paraplegia (HSP) in whom they identified a homozygous c.772G $>$ A (p.Glu258Lys) variant in the $T H$ gene, which they classified as possibly causative. This suggested that the TH gene, known to be responsible for autosomal recessive dopamine-responsive dystonia, could also be associated with HSP, as has been suggested for $\mathrm{GCH} 1$, the gene for autosomal dominant dopamine-responsive dystonia. ${ }^{1}$ However, recently mutations in CAPN1 were found in a new form of autosomal recessive HSP (SPG78) ${ }^{2}$ and querying their exome data revealed a homozygous nonsense mutation (Chr11 (GRCh37):g.64951004C > T; NM_005186.3:c.397C > T; p.(Arg133*)) in CAPN1 in this particular sibship. The authors believe that this is the more likely cause for the autosomal recessive HSP in this family.

\section{Mitochondrial encephalomyopathy and retinoblastoma explained by compound heterozygosity of SUCLA2 point mutation and $13 q 14$ deletion}

Sanna Matilainen, Pirjo Isohanni, Liliya Euro, Tuula Lönnqvist, Helena Pihko, Tero Kivelä, Sakari Knuutila and Anu Suomalainen

European Journal of Human Genetics (2017) 25, 393; doi:10.1038/ejhg.2016.166

Correction to: European Journal of Human Genetics (2015) 23, 325-330; doi:10.1038/ejhg.2014.128

Previously reported patients (22)

Patient 1

Patient 2

The sub-heading of Table 1 is incorrect and should read: 